\title{
Do we need to take care of nodular scar following gastric ESD?
}

\section{(ㄷ)(1) $\odot$}

\author{
Authors \\ Motohiko Kato, Naohisa Yahagi \\ Institution \\ Division of Research and Development for Minimally \\ Invasive Treatment, Cancer Center, Keio University School \\ of Medicine, Tokyo, Japan \\ Bibliography \\ DOI https://doi.org/10.1055/a-0608-4329 | \\ Endoscopy International Open 2018; 06: E1204-E1205 \\ (c) Georg Thieme Verlag KG Stuttgart · New York \\ ISSN 2364-3722
}

\author{
Corresponding author \\ Motohiko Kato, MD, PhD, Keio University School of \\ Medicine - Cancer Center, 35 Shinanomachi, Shinjuku-ku \\ Tokyo - Select One - 160-8582, Japan \\ Fax: +81-3-3353-6247 \\ moto28hiko@icloud.com
}

Piecemeal resection and a positive or unclear horizontal margin is known to be associated with risk of local recurrence. Incidence rate of local residual recurrence after incomplete endoscopic resection is reported to be more than $20 \%$ to $30 \%$ [ 1 , 2]. Endoscopic submucosal dissection (ESD) achieves en bloc resection and $\mathrm{R} 0$ resection in over $90 \%$ of cases $[3,4]$. This high success rate contributes to a significant reduction in local residual recurrence. In fact, incidence rate oflocal recurrence after curative resection is reportedly extremely low $[1,5,6]$. On the other hand, we sometimes encounter polypoid nodules on the ESD scar even though pathological findings show the initial ESD resulted in complete resection.

In this issue of Endoscopy International Open, Arantes et al. demonstrated the results of a multicenter retrospective study of polypoid nodule scars (PNSs) after gastric ESD. They analyzed a total of 2275 curative gastric ESD cases from 5 referral centers and found that incidence of PNS was $1.2 \%$ (3.1\% in the distal stomach), all cases of PNS arose from the distal stomach, and approximately $20 \%$ of PNS disappeared during follow-up. Based on these results, they concluded that PNS is a benign alteration of wound healing and does not require any intervention other than endoscopic surveillance.

ESD as a salvage treatment for local residual recurrence after endoscopic treatment is a technically difficult procedure due to the severe fibrosis in the submucosal layer from the wound healing process. In addition, there is no space to dissect, particularly after ESD in cases in which the dissection layer is just above the muscularis propria. Severe fibrosis results in poor visualization of the submucosal layer and increased difficulty in identification of the dissecting plane; thus, ESD is associated with an increased risk of perforation and incomplete resection due to injury of the specimen. Considering the difficulty of ESC, a precise diagnosis to differentiate between PNS and true local recurrence is important.

The precise reason PNS predominantly arises from the distal stomach is unknown. The gastric antrum is a site of predilection for inflammatory fibroid polyps, which are non-neoplastic celIular proliferations composed of fibroblasts, blood vessels, and inflammatory cells [7]. This observation suggests the inflammatory reaction and wound healing process could alternate between the antrum and the fundus/body. Differences in the properties of gastric peristalsis, the degree of bile reflux, and the thickness of the submucosal layer according to the location would play a role in developing PNS.

\section{Conclusion}

In conclusion, to avoid an unnecessary salvage ESD, we should consider PNS, a non-neoplastic change, as a possibility, especially when we find a polypoid change on the ESD scar in the distal stomach.

\section{Competing interests}

None 


\section{References}

[1] Hoteya S, lizuka T, Kikuchi D et al. Secondary endoscopic submucosal dissection for residual or recurrent tumors after gastric endoscopic submucosal dissection. Gastric Cancer 2014; 17: 697 - 702

[2] Horiki N, Omata F, Uemura M et al. Risk for local recurrence of early gastric cancer treated with piecemeal endoscopic mucosal resection during a 10-year follow-up period. Surg Endosc 2012; 26: 72 - 78

[3] Oda I, Gotoda T, Hamanaka H et al. Endoscopic submucosal dissection for early gastric cancer: technical feasibility, operation time and complications from a large consecutive series. Digest Endosc 2005; 17: $54-58$

[4] Kato M, Nishida T, Tsutsui S et al. Endoscopic submucosal dissection as a treatment for gastric noninvasive neoplasia: a multicenter study by Osaka University ESD Study Group. J Gastroenterol 2011; 46: 325331

[5] Kato M, Nishida T, Yamamoto K et al. Scheduled endoscopic surveillance controls secondary cancer after curative endoscopic resection for early gastric cancer: a multicentre retrospective cohort study by Osaka University ESD study group. Gut 2013; 62: 1425 - 1432

[6] Isomoto H, Shikuwa S, Yamaguchi $\mathrm{N}$ et al. Endoscopic submucosal dissection for early gastric cancer: a large-scale feasibility study. Gut 2009; 58: $331-336$

[7] Harned RK, Buck JL, Shekitka KM. Inflammatory fibroid polyps of the gastrointestinal tract: radiologic evaluation. Radiology 1992; 182: $863-866$ 